PROCEEDINGS OF THE

AMERICAN MATHEMATICAL SOCIETY

Volume 135, Number 4, April 2007, Pages 1059-1063

S 0002-9939(06)08536-4

Article electronically published on September 18, 2006

\title{
ON BANACH LATTICES WITH LEVI NORMS
}

\author{
BIROL ALTIN
}

(Communicated by N. Tomczak-Jaegermann)

\begin{abstract}
Schmidt proved that an operator $T$ from a Banach lattice $E$ into a Banach lattice $G$ with property $(P)$ is order bounded if and only if its adjoint is order bounded, and in this case $T$ satisfies $\||T|\|=\left\|\left|T^{\prime}\right|\right\|$. In the present paper the result is generalized to Banach lattices with Levi-Fatou norm serving as range, and some characterizations of Banach lattices with a Levi norm are given. Moreover, some characterizations of Riesz spaces having property $(b)$ are also obtained.
\end{abstract}

\section{INTRODUCTION AND PRELIMINARIES}

The order duals $E^{\sim}$ and $E^{\sim \sim}$ of $E$ are Riesz spaces whenever $E$ is a Riesz space. It is well known that the canonical embedding $Q_{E}: E \rightarrow E^{\sim \sim}$ defined by $Q_{E}(x)=\hat{x} ; \hat{x}(f)=f(x), f \in E^{\sim}, x \in E$ is a lattice homomorphism. If $E^{\sim}$ separates the points of $E$, then $Q_{E}$ is also one-to-one, and hence $E$ can be considered as a Riesz subspace of $E^{\sim \sim}$. We will assume all Riesz spaces considered in this note have seperating order duals.

Recall that a Banach lattice $E$ is said to have property $(P)$ if there exists a positive contractive projection $P: E^{\prime \prime} \rightarrow E$. Property $(P)$ implies that $E$ is Dedekind complete. Clearly, every KB-space has property $(P)$. Also every Dedekind complete AM-space with unit has property $(P)$. A norm on a Banach lattice is said to be a Levi norm if every norm bounded upward directed set of positive elements has a supremum. Obviously, each Banach lattice with a Levi norm must be Dedekind complete. A norm on a Banach lattice $E$ is said to be a Fatou norm if $0 \leq x_{\alpha} \uparrow x$ in $E$ implies $\left\|x_{\alpha}\right\| \uparrow\|x\|$.

In all undefined terminology concerning Riesz spaces we will adhere to 2 and [3].

\section{Some Characterizations of Banach lattices With LeVi norms}

The following proposition generalizes Lemma 2.1 in [7] since every Banach lattice with property $(P)$ has a Levi-Fatou norm.

Proposition 1. Let $E$ and $F$ be two Banach lattices with $F$ having a Levi norm. Then a continuous operator $T: E \rightarrow F$ is order bounded if and only if its adjoint

Received by the editors January 25, 2005 and, in revised form, June 22, 2005, July 18, 2005, and October 25, 2005.

2000 Mathematics Subject Classification. Primary 46A40; Secondary 47B65.

Key words and phrases. Banach lattices, Levi norm, property (b), regular norm.

(C)2006 American Mathematical Society 
$T^{\prime}: F^{\prime} \rightarrow E^{\prime}$ is order bounded. In particular, if $F$ also has a Fatou norm, then $T$ satisfies $\||T|\|=\left\|\left|T^{\prime}\right|\right\|$.

Proof. Let $T: E \rightarrow F$ be an order bounded operator, $f \in\left(F^{\prime}\right)^{+}, g \in F^{\prime}$ with $|g| \leq f$. Then we have $\left|T^{\prime} g\right|=|g T| \leq|g||T| \leq f|T|=|T|^{\prime} f$. Therefore, $T^{\prime}$ is order bounded.

For the converse, assume that $T^{\prime}$ is an order bounded operator. Hence, $T^{\prime \prime}$ is order bounded. Now let $x \in E^{+}, y \in E$ with $|y| \leq x$; we have $|T(y)|=\left|T^{\prime \prime}(y)\right| \leq$ $\left|T^{\prime \prime}\right|(x)$. Since $F$ has a Levi norm, there exists a function $\varphi: F^{\prime \prime} \rightarrow F$ defined by $\varphi\left(x^{\prime \prime}\right)=\sup \left\{\left[0,\left|x^{\prime \prime}\right|\right] \cap F\right\}$ satisfying the following conditions:

(1) For every element $x^{\prime \prime}, y^{\prime \prime} \in F^{\prime \prime}$ with $\left|x^{\prime \prime}\right| \leq\left|y^{\prime \prime}\right|, 0 \leq \varphi\left(x^{\prime \prime}\right) \leq \varphi\left(y^{\prime \prime}\right)$.

(2) For each element $x$ of $F^{+}, \varphi(x)=x$.

So, we have $|T(y)|=\varphi\left(\left|T^{\prime \prime}(y)\right|\right) \leq \varphi\left|T^{\prime \prime}\right|(x) \leq \varphi\left|T^{\prime}\right|^{\prime}(x)$. Hence, $T$ is order bounded. Also, from Theorem 1.10 in [2] we see that $|T| x) \leq \varphi\left|T^{\prime}\right|^{\prime}(x)$ for all $x \in E^{+}$.

Now, let $F$ have a Levi-Fatou norm, let $x^{\prime \prime} \in F^{\prime \prime}$ and let $x_{\alpha}=\alpha$ for each $\alpha \in\left[0,\left|x^{\prime \prime}\right|\right] \cap F$. By the definition of $\varphi$ we have $0 \leq x_{\alpha} \uparrow \varphi\left(x^{\prime \prime}\right)$ in $F$. Since the norm on $F$ is Fatou and $\left\|x_{\alpha}\right\| \leq\left\|x^{\prime \prime}\right\|$ for each $\alpha$, we have $\left\|\varphi\left(x^{\prime \prime}\right)\right\| \leq\left\|x^{\prime \prime}\right\|$. Therefore, $\||T|(x)\| \leq\left\|\varphi\left|T^{\prime}\right|^{\prime}(x)\right\| \leq\left\|\left|T^{\prime}\right|^{\prime}(x)\right\| \leq\left\|\left|T^{\prime}\right|^{\prime}\right\|\|x\|=\left\|\left|T^{\prime}\right|\right\|\|x\| \leq$ $\left\||T|^{\prime}\right\|\|x\|=\||T|\|\|x\|$. Hence, $\||T|(x)\| \leq\left\|\left|T^{\prime}\right|\right\|\|x\| \leq\||T|\|\|x\|$. This implies that $\||T|\|=\left\|\left|T^{\prime}\right|\right\|$.

The preceding proposition coupled with Theorem 3.5 in 1 yields the following characterization.

Corollary 1. Let $F$ be a Banach lattice with Dedekind complete. Then the following statements are equivalent:

(a) F has a Levi norm.

(b) For every Banach lattice $E$, if $T^{\prime} \in L_{b}\left(F^{\prime}, E^{\prime}\right)$ when $T \in L(E, F)$, then $T \in L_{b}(E, F)$.

Let $E$ be a Banach lattice and let $x^{\prime \prime}$ be a positive element of $E^{\prime \prime}$. Let $I_{x^{\prime \prime}}$ denote the principal ideal generated by $x^{\prime \prime}$ in $E^{\prime \prime}$ and let $E_{x^{\prime \prime}}$ denote the Riesz space $I_{x^{\prime \prime}} \cap E$. It is known that $E_{x^{\prime \prime}}$ is an AM-space with the norm

$$
\|u\|_{x^{\prime \prime}}=\inf \left\{\lambda>0:|u| \leq \lambda x^{\prime \prime}\right\}, u \in E_{x^{\prime \prime}} .
$$

The proof of the following proposition is straightforward and is therefore omitted.

Proposition 2. Let $E$ be a Banach lattice with an order continuous norm. Then $E$ has a Levi norm (i.e., $E$ is a KB-space) if and only if the norm $\|\cdot\|_{x^{\prime \prime}}$ on the Banach lattice $E_{x^{\prime \prime}}$ is a Levi norm for each $x^{\prime \prime} \in\left(E^{\prime \prime}\right)^{+}$.

Let $\Omega$ be the collection of all norm bounded upward directed sets of positive elements of $E$. Then for each $A \in \Omega$ the formula

$$
\rho_{A}\left(x^{\prime}\right)=\sup \left\{\left|x^{\prime}\right|(x): x \in A\right\}, x^{\prime} \in E^{\prime},
$$

defines a Riesz seminorm on $E^{\prime}$, and thus, $\Omega$ generates a locally convex-solid topology on $E^{\prime}$ via the family of seminorms $\left\{\rho_{A}: A \in \Omega\right\}$. This topology is denoted by $\left|\sigma_{l}\right|\left(E^{\prime}, E\right)$.

It is clear that the well-known absolute weak topology $|\sigma|\left(E^{\prime}, E\right)$ on $E^{\prime}$ is coarser than $\left|\sigma_{l}\right|\left(E^{\prime}, E\right)$ (i.e., $\left.|\sigma|\left(E^{\prime}, E\right) \subseteq\left|\sigma_{l}\right|\left(E^{\prime}, E\right)\right)$. 
The following proposition shows that the inclusion $|\sigma|\left(E^{\prime}, E\right) \subseteq\left|\sigma_{l}\right|\left(E^{\prime}, E\right)$ is proper when $E$ does not have any Levi norm.

Proposition 3. Let $E$ be a Dedekind complete Banach lattice. Then $E$ has a Levi norm if and only if $|\sigma|\left(E^{\prime}, E\right)=\left|\sigma_{l}\right|\left(E^{\prime}, E\right)$.

Proof. Let $E$ have a Levi norm. Then it is clear that $|\sigma|\left(E^{\prime}, E\right)=\left|\sigma_{l}\right|\left(E^{\prime}, E\right)$.

To prove the converse, suppose that the equality $|\sigma|\left(E^{\prime}, E\right)=\left|\sigma_{l}\right|\left(E^{\prime}, E\right)$ is satisfied and $\left\{x_{\alpha}\right\}$ is an increasing, norm-bounded net in $E^{+}$. Then $A=\left\{x_{\alpha}: \alpha \in\right.$ $\Lambda\}$ is order bounded in $E^{\prime \prime}$. By Theorem 11.11 in [2], there exists an element $x$ of $E^{+}$with $[-x, x]^{o} \subseteq\left\{x^{\prime} \in E^{\prime}: \rho_{A}\left(x^{\prime}\right) \leq 1\right\} \subseteq A^{\circ}$ in $E^{\prime}$. By the Bipolar Theorem, we have $[-x, x]^{\circ \circ}=[-x, x]$. It follows that $A \subseteq[-x, x]$ and $E$ has a Levi norm.

\section{Some characterizations of Riesz spaces With Property $(b)$}

Let $E$ be a Riesz space and let $A$ be a subset of $E$. If $A$ is order bounded in $E^{\sim \sim}$, then $A$ is said to be $b$-order bounded in $E$. A Riesz space $E$ is said to have property $(b)$ if every $b$-order bounded subset of $E$ is order bounded in $E$ [4. Every order dual and perfect Riesz space has property $(b)$; if $E^{\sim \sim}$ retractable on $E$, then $E$ has property $(b)$, and for an arbitrary compact Hausdorff space $K, C(K)$ has property $(b)$. But, $c_{0}$ does not have the property $(b) 4$. The Levi property and Zaanen's $B$-property also imply property $(b)$ in Frechet lattices. However, $C[0,1]$, with the supremum norm, has neither the Levi property nor the $B$-property [4].

A Riesz space $E$ has property $(b)$ if and only if for each net $\left\{x_{\alpha}\right\}$ in $E$ satisfying $0 \leq x_{\alpha} \uparrow x^{\prime \prime}$ for some $x^{\prime \prime}$ in $E^{\sim \sim},\left\{x_{\alpha}\right\}$ is order bounded in $E$ [4.

In [5], it is seen that $\mathcal{L}_{b}(E, F)$ has property $(b)$ if and only if $F$ has property $(b)$, where both $E$ and $F$ are two Banach lattices and $F$ is Dedekind complete. The following proposition generalizes the result to the case of Riesz spaces.

Proposition 4. If $E$ and $F$ are two Riesz spaces with $F$ Dedekind complete, then $\mathcal{L}_{b}(E, F)$ has property $(b)$ if and only if $F$ has property $(b)$.

Proof. Assume first that $\mathcal{L}_{b}(E, F)$ has property $(b)$. Take a net $\left\{y_{\alpha}\right\}$ in $F$ with $0 \leq y_{\alpha} \uparrow y^{\prime \prime}$ in $F^{\sim \sim}$. Then we choose $f \in\left(E^{\sim}\right)^{+}$with $f \neq 0$. Define $\phi: F \rightarrow$ $\mathcal{L}_{b}(E, F), y \rightarrow f \otimes y,(f \otimes y)(x)=f(x) . y$ for each $x \in E$. It is known that $\phi$ is a Riesz isomorphism. Since $\left\{y_{\alpha}\right\}$ is $b$-order bounded in $F, \phi\left(y_{\alpha}\right)$ is $b$-order bounded in $\mathcal{L}_{b}(E, F)$. From the hypothesis there exists $T$ in $\mathcal{L}_{b}(E, F)$ such that $0 \leq f \otimes y_{\alpha} \leq T$. Hence, there exists a positive element $x_{o}$ in $E$ such that $0 \leq y_{\alpha} \leq T\left(x_{o}\right)$ in $F$. Thus $F$ has property $(b)$.

Suppose now $F$ has property $(b)$ and $\left(T_{\alpha}\right)$ is a net in $\mathcal{L}_{b}(E, F)$ with $0 \leq T_{\alpha} \uparrow T^{* *}$ in $\left[\mathcal{L}_{b}(E, F)\right]^{\sim \sim}$. We choose $x \in E^{+}$with $x \neq 0$. Define $\psi: F^{\sim} \rightarrow \bar{L}_{b}(E, F)^{\sim}$, $f \rightarrow \psi(f), \psi(f)(T)=f(T x), T \in \mathcal{L}_{b}(E, F)$. Clearly $\psi$ is one-to-one and positive. Therefore, we have $0 \leq f\left(T_{\alpha}(x)\right) \leq T^{* *}(\psi(f))$, for each $f \in\left(F^{\sim}\right)^{+}$. Thus, $\left\{T_{\alpha}(x)\right\}$ is a $b$-order bounded set in $F$ for each $x \in E^{+}$. From the hypothesis there exists $y(x) \in F$ such that $0 \leq T_{\alpha}(x) \uparrow y(x)$ for each $x \in E^{+}$. The mapping $T: E^{+} \rightarrow F^{+}$ defined by $T(x)=\sup T_{\alpha}(x)$ is additive on $E^{+}$. We can extend $T$ to a unique positive operator on $E$. Clearly $0 \leq T_{\alpha} \leq T$, and so $\mathcal{L}_{b}(E, F)$ has property $(b)$.

Furthermore, the following question, "when does the Dedekind completion of a Riesz space have property $(b)$ ?" is taken into consideration.

Proposition 5. Let $E$ be a Riesz space. Then $E$ has property (b) if and only if Dedekind completion of $E$ has property $(b)$. 
Proof. Let $E$ have property $(b)$ and let $E^{\delta}$ be a Dedekind completion of $E$. By Theorem 7.12 in [2], $E$ is Riesz isomorphic to a Riesz subspace of $E^{\delta}$ such that for each $x \in E^{\delta}$ we have

$$
x=\sup \{y \in E: y \leq x\}=\inf \{z \in E: x \leq z\},
$$

given a net $\left\{x_{\alpha}^{\delta}\right\}$ in $E^{\delta}$ and given $0 \leq x^{\prime \prime} \in\left(E^{\delta}\right)^{\sim}$ such that $0 \leq x_{\alpha}^{\delta} \uparrow \leq x^{\prime \prime}$ in $\left(E^{\delta}\right)^{\sim \sim}$. Thus, there exists a net $\left\{x_{\beta(\alpha)}\right\} \subset E$ such that $0 \leq x_{\beta(\alpha)} \uparrow_{\beta(\alpha)} x_{\alpha}^{\delta} \leq x^{\prime \prime}$ in $\left(E^{\delta}\right)^{\sim \sim}$ for each $\alpha$. Therefore, we have $0 \leq f\left(x_{\beta(\alpha)}\right) \leq x^{\prime \prime}(f)$ for each $f \in$ $\left(\left(E^{\delta}\right)^{\sim}\right)^{+}$. By Theorem 2.8 in [2] for each $0 \leq f \in E^{\sim}$, there exists an extension $\hat{f}$ from $E^{\delta}$ into $\mathbb{R}$. Consequently, we have $0 \leq f\left(x_{\beta(\alpha)}\right) \leq \hat{f}\left(x_{\alpha}^{\delta}\right) \leq x^{\prime \prime}(\hat{f})$. Hence, the set $\left\{x_{\beta(\alpha)}\right\}$ is $b$-order bounded in $E$. Thus, by hypothesis, there exists $x$ in $E$ such that $0 \leq x_{\beta(\alpha)} \leq x$ for all $\alpha, \beta(\alpha)$. So, we have $0 \leq x_{\alpha}^{\delta} \leq x$. Therefore, $E^{\delta}$ has property $(b)$.

To prove the converse, suppose that $E^{\delta}$ has property $(b)$. Let $\left\{x_{\alpha}\right\}$ be a net in $E$ with $0 \leq x_{\alpha} \uparrow \leq x^{\prime \prime}$ in $E^{\sim \sim}$. Define the functional $\hat{x}:\left(E^{\delta}\right)^{\sim} \rightarrow \mathbb{R}$ by $\hat{x}: f \rightarrow$ $x^{\prime \prime}\left(f_{\mid E}\right)$. Then we have $0 \leq x_{\alpha} \uparrow \leq \hat{x}$ in $\left(E^{\delta}\right) \sim \sim$. Thus, there exists a positive element $z$ in $E$ with $0 \leq x_{\alpha} \leq z$. Hence, $E$ has property $(b)$, as desired.

Let $\left\{E_{i}: i \in I\right\}$ be a family of Riesz spaces. Then the cartesian product $\prod_{i \in I} E_{i}$, under the ordering $\left\{x_{i}\right\} \leq\left\{y_{i}\right\}$ whenever $x_{i} \leq y_{i}$ holds for all $i \in I$, is a Riesz space. The direct sum $\sum_{i \in I} \bigoplus E_{i}$ is the vector space of all elements $\left\{x_{i}\right\}$ of $\prod_{i \in I} E_{i}$ for which $x_{i}=0$ holds for all but a finite number of $i . \sum_{i \in I} \bigoplus E_{i}$ is a Riesz subspace of $\prod_{i \in I} E_{i}$ with the pointwise algebraic and lattice operations [2]. The next proposition characterizes the direct sum $\sum_{i \in I} \bigoplus E_{i}$ with property $(b)$.

Proposition 6. Let $\left\{E_{i}: i \in I\right\}$ be a family of Riesz spaces. The direct sum $\sum_{i \in I} \oplus E_{i}$ has property (b) if and only if each $E_{i}$ has property $(b)$.

Proof. Let $E_{i}$ have property (b) for each $i \in I$, given a net $\left\{x_{\alpha}=\left(x_{\alpha}^{i}\right), x_{\alpha}^{i} \in E_{i}\right.$ for each $i \in I, \alpha \in \Lambda\}$ in $\sum_{i \in I} \bigoplus E_{i}$ with $0 \leq x_{\alpha} \uparrow \leq x^{\prime \prime}$ in $\left(\sum_{i \in I} \bigoplus E_{i}\right)^{\sim \sim}$. It is enough to show that there exists a finite subset $J$ of $I$ such that $x_{\alpha}^{i}=0$ for all $\alpha$ and $i \in I \backslash J$. To see this, assume by way of contradiction that there exist $\alpha_{n} \in \Lambda$ and $i_{n} \in I$ such that $x_{\alpha_{n}}^{i_{n}} \neq 0$ for each $n \in \mathbb{N}$. One can choose $\alpha_{n} \in \Lambda$ with $\alpha_{n} \leq \alpha_{n+1}$ for each $n \in \mathbb{N}$. Since $E_{i_{n}}^{\sim}$ separates the points of $E_{i_{n}}$ for each $i_{n}$, then there exists $g_{i_{n}} \in\left(E_{i_{n}}^{\sim}\right)^{+}$with $g_{i_{n}}\left(x_{\alpha_{n}}^{i_{n}}\right)=1$. Now define a functional $g$ :

$$
g=\left(g_{i}\right)= \begin{cases}g_{i_{n}}, & \text { if there exists an } n \in \mathbb{N} \text { such that } i=i_{n}, \\ 0, & \text { otherwise. }\end{cases}
$$

It is clear that $g \in \prod_{i \in I} E_{i}^{\sim}$. On the other hand, the operator $\varphi: \prod_{i \in I} E_{i}^{\sim} \rightarrow$ $\left(\sum_{i \in I} \oplus E_{i}\right)^{\sim}$, defined by $\varphi(f)=\hat{f}, \hat{f}(x)=\sum_{i \in I} f_{i}\left(x_{i}\right), f=\left(f_{i}\right) \in \prod_{i \in I} E_{i}^{\sim}$ and $x=\left(x_{i}\right) \in \sum_{i \in I} \bigoplus E_{i}$, is a positive operator. Therefore, $0 \leq \sum_{i \in I} g_{i}\left(x_{\alpha}^{i}\right) \leq$ $x^{\prime \prime}(\varphi(g))$ for each $\alpha \in \Lambda$ and $i \in I$. Hence, we see that $n \leq \sum_{i \in I} g_{i}\left(x_{\alpha}^{i}\right) \leq x^{\prime \prime}(\varphi(g))$ for each $n$, which is impossible. Thus, $\sum_{i \in I} \bigoplus E_{i}$ has property $(b)$.

The converse is routine and is omitted.

\section{REFERENCES}

[1] Y. A. Abramovich and A. W. Wickstead, When each continuous operator is regular, II, Indag. Math., 8, 1997, 281-294. MR.1622216 (99c:47046) 
[2] C. D. Aliprantis and O. Burkinshaw, Positive Operators, Academic Press, New York and London (1985). MR0809372 (87h:47086)

[3] C. D. Aliprantis and O. Burkinshaw, Locally Solid Riesz Spaces, Academic Press, New York, San Francisko and London (1978). MR0493242 (58:12271)

[4] Ş. Alpay, B. Altın and C. Tonyal, On property (b) of vector lattices, Positivity, 7, 2003, 135-139. MR2028377(2005a:46007)

[5] Ş. Alpay, B. Altın and C. Tonyalı, A note on Riesz spaces with property-b, Czech. Math. J. (to appear).

[6] P. Meyer-Nieberg, Banach lattices, Springer-Verlag, Berlin, Heidelberg, New York (1991). MR.1128093 (93f:46025)

[7] K. D. Schmidt, On the modulus of weakly compact operators and strongly additive vector measures, Proc. Amer. Math. Soc., 102, 1988, 862-866. MR0934857 (89e:47056)

Department of Mathematics, Faculty of Science and Arts, Gazi University, 06500, Teknikokullar, Ankara, Turkey

E-mail address: birola@gazi.edu.tr 